\title{
ON HYDROGEN AND ENERGY SYSTEMS
}

Reprint

C. Marchetti

\author{
RR-76-7 \\ March 1976
}

Research Reports provide the formal record of research conducted by the International Institute for Applied Systems Analysis. They are carefully reviewed before publication and represent, in the Institute's best judgment, competent scientific work. Views or opinions expressed therein, however, do not necescarily reflect those of the National Member Organizations supporting the Institute or of the Institute itself. 



\section{PREFACE}

This paper was invited by Pergamon Press to inaugurate its new journal dedicated to Hydrogen Energy.

The background thesis is that the physical properties of the energy vectors have a preponderant effect on the evolution of energy systems and of the mix of primary energies. Within the frame and the limitations of the arguments presented it appears that hydrogen represents the best energy vector in a broad range of boundary conditions, and that hydrogen-based energy transportation and distribution systems are the most likely to prevail in the long run.

This kind of analysis is based on some of the results of the IIASA energy group studies on primary energy substitution and transitions. 



\title{
ON HYDROGEN AND ENERGY SYSTEMS
}

\author{
C. MARChETTI \\ International Institute for Applied Systems Analysis* Laxenburg, Austria
}

\begin{abstract}
INTRODUCTION
THE RUTHLESS maneuvering of the oilmen. and the panic generated by skillful embargos and great increases in oil prices has focused the attention of politicians, scientists and laymen on the problem of resources and scarcity.

In the case of energy, looking at the objective situation one has the feeling that the circus has gone a little too far. If resources per se are really the controlling factor, let us examine the most trivial case, that of primary productivity of world forests. After all. wood has been for thousands of years the main source of energy. Well, with a total energy consumption by humanity of around 7 Terawatts (roughly $7 \times 10^{9}$ Tons coal equivalent/year) the primary production of wood in the forests is about $40 \mathrm{TW}$ or seven times larger. [1] And fully renewable!

If one likes big figures, then one had the $300,000 \mathrm{TW}$ of solar energy flowing over the earth, and thermodynamically almost as good as mechanical energy if the earth surface temperature is taken as reference for the "cold source". As this energy is finally flowing into the outer space, whose temperature can be taken at $-3^{\circ} \mathrm{K}$, one can play with the idea of considering this as the final sink, in which case the solar flux is worth a hundred times more.

On the other side. if one looks at the past history (Fig. 1) one sees that man starts shifting from one primary energy to another long before the exhaustion of any particular kind of primary resource. This is clear for wood, and obvious for coal. We shall not enter into discussing oil and gas because the demonstration is more complicated.

This introduction has the scope to move the spotlight from the resources to the energy systems. i.e. that complex gadgetry sitting between the primary resource and the final consumer. It is there where the eventual causes of malfunctioning are hidden. and where the cures have to be applied.
\end{abstract}

\section{ON ENERGY SYSTEMS}

Looking again at Fig. 1, one can gather some very general and important information about the aggregated functioning of these systems. Their behavior appears to be strictly analogous to that of any other industrial-commercial enterprise. where new products or processes are slowly substituting old ones [1, 2] (Figs. 2). The substitution follows quite strictly a logistic pattern even if the substitution takes a century or so (as many do). This gives a high degree of predictability to these substitutions. once initiated and well established, and in particular in the energy case the primadonna role for the next half century or so, seems to be assured for natural gas (Fig. 3).

Where all this gas will come from would again require a long discussion. Resources do not appear to be the constraint, but an economical Liquid Natural Gas (LNG) chain. The problem being primarily technological it will be very probably satisfactorily solved. and we assume to be so. This means a large infrastructure will be developed for transportation and distribution of a gaseous fuel, irradiating mainly from the ocean LNG terminals. This infrastructure will be a most precious asset for any new compatible gaseous fuel that may appear on the market.

Coming back to our penetration curves, the next round secms to be in the hand of nuclear energy. It already has a foot in the door, and in spite of the numerous and inevitable teething troubles one can expect it will finally be allowed in. Solar, fusion and miscellaneous are still on the bench, and the enormous lead times for introducing new

t Any views or conclusions are those of the author. and do not necessarily reflect those of IIASA. 


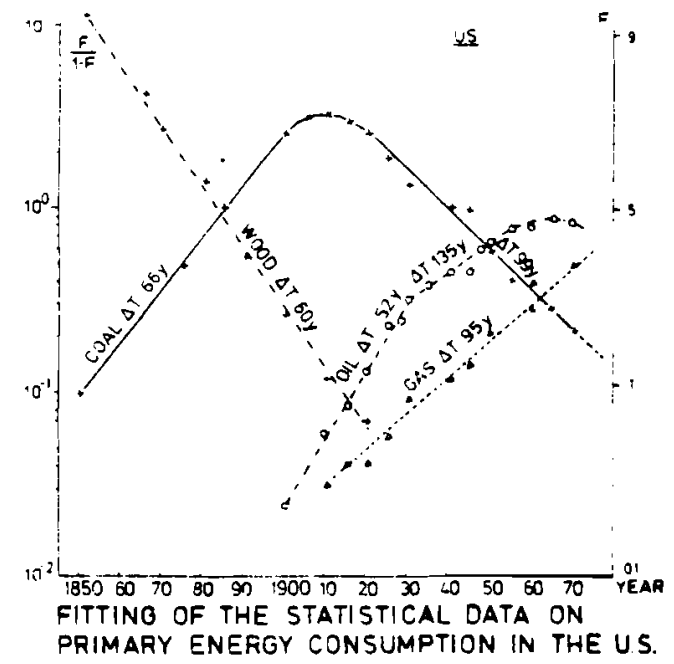

FIG. 1. Fitting of statistical data for energy consumption in U.S. From Ref. 3. $F$ is the fraction of the market covered by a paricular primary fuel. measured in energy units. $\Delta T$ indicates the time for a given fuel to go from $1 \%$ of the market to $50 \%$ and represents a kind of time constant for the substitution process.

Substitutions in energy never go complete due to the appearance on the market of new competitors (From Ref. 3).

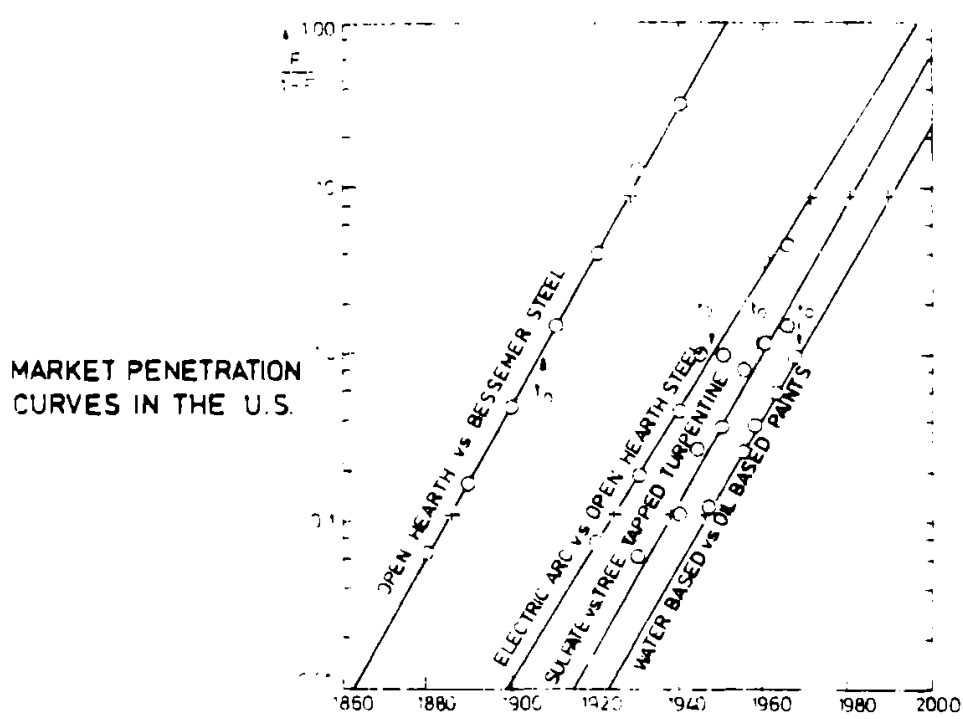

Fig. 2a. Miscellaneous examples of substitutions in the U.S. market in substitution of other preexisting processes $(l-F)$. The function describing the substitution is in $(F / 1-F)=a t+c$ where $t$ is time and $\alpha, c$ empirical constants (From Ref. 1). 
PENETRATION OF BOF VS. OPEN HEARTH AND BESSEMER STEEL

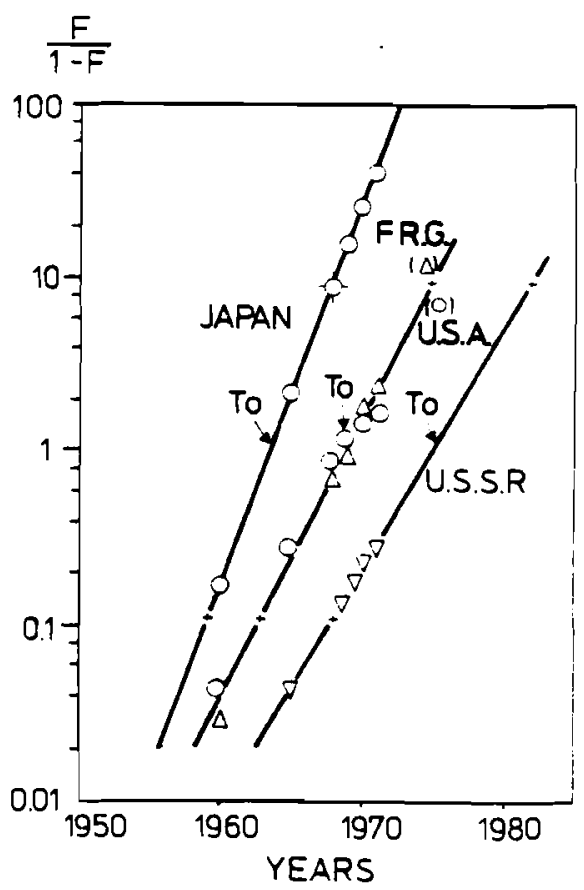

FIG 2b. The introduction of the Basic Oxygen Furnace process to produce steel in substitution of other preexisting processes (From Ref. 2).

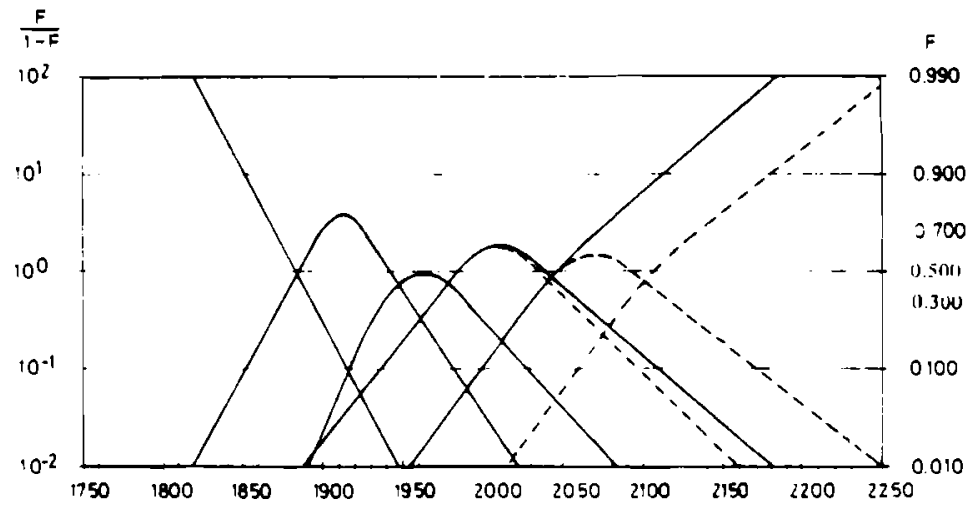

FIG. 3. Sequence as in Fig. 2. The last curves hypothize a new energy source making its inroads at the beginning of the next century (Fusion?) and its effect on gas and nuclear market share. The others are not affected (From Ref. 3). Curves refer to U.S. 
technologies on such a large scale, well exemplified by the case of nuclear energy, make them a good argument for the year 2000. To keep the scale in mind, at current oil prices the $7 \mathrm{TW}$ humanity consumes, are worth $500 \times 10^{\circ} \$ /$ year. and investments for new energy sources have to be looked at in this context.

So we are left with a very probable nuclear development as penetration curves seem to never come back after $F>2-3 \%$, a very probable gas fuel infrastructure and a very complex problem to untwist: what is the most economic way to move ahead respecting the numerous constraints.

As we said at the beginning, the energy system makes the history, and not the energy source. But what makes the energy system run? We think we have identified the critical parameters. in the transportability and storability of the fuel or energy vector. and in the economies of scale of the interfaces and transformation points [4].

The electrical energy system serves well the purpose of illustrating the point. The cost of a power station growing roughly as the two-third power of its capacity, there is a strong incentive to build big and concentrate generation. On the other hand, transportation costs and the necessity to provide a standby capacity sufficient to take care for the accidental outage of a power station, pushes toward decentralized generation and small size. The actual structure of an electric net results from the interaction of these three parameters.

Taking evolution in voltage for the power lines as an indicator for the mean distance at which electric energy is transported, we find this distance to double every 22-23 years. Consumption during the last 80 years did increase quite steadily with a doubling time of 9-10 years or so. Combining the two growth rates. one finds a doubling time for the power controlled by a net of 6-7 years. Not by chance this has been the doubling time of the capacity of the electric generators and associated steam turbines which have doubled in size every 6.5 years through five order of magnitude!

The example is obviously very simplified. but it still carries a central message: the transportability of the energy vector or of the fuel. plays a substantial role in determining the scale and the economy of the operation.

As a kind of counterproof. in Fig. + a historical case is reported. That of a wystem of mechanical energy transport based in steel cables built in Schaffhausen 100 years ago. Although very well ingineered, and reasonably efticient. it was doomed by the fact that it could curry energy only a few hundred meters away.

ln an electric system. energy is transported today at a mean distance of about $100 \mathrm{~km}$. in a natural gals svistem (e.g. in L.S.) it can well be at $1000 \mathrm{~km}$.

For the oil system. the supertankers make it a world system. The analogy with the electric grid says if it could be optimized from scratch. a dozen major oil fields or regions would realize the hest contiguration and that's what happened in fact. obviously by chance. In any case. this analogy should be considered only heuristically, as the economies of scale for power stations and oil fielus have different scale factors.

For the natural gas. the $1000 \mathrm{~km}$ of economic transport with the trunkline give the system a continental or subcontinental scale. It is already so in Europe, Soviet Union and United States. Sea transportation of natural gas using cryotankers started developing only in the last decade. Up to now. natural gas so precious and convenient at the level of the consumer was considered a nuisance at the level of the oil producer if a pipeline grid was not at hand. and usually flared. When dry-gas fields were discovered they were plugged back as valueless. This again stresses the dominance of the intermediate system over the physical resources.

\section{ON HYDROGEN}

The economics of hydrogen transportation over a pipeline are very parallel to that of natural gas of that we may safely assume the same system characteristics for the two. Furthermore all fixed utilizations could easily switch from natural gas to hydrogen with some modifications in the burners as a vast literature indicates [5]. Hydrogen appears then the natural heir of natural gas. sitisfact-rily equipped to slip into the system developed for, which by all means will outlive the inevitable if slow exhaustion of the gas fields. This is exactly what natural gas has done to the town-gms systems of Europe which was distributing gaseous products from coal, i.e. a mixture of 


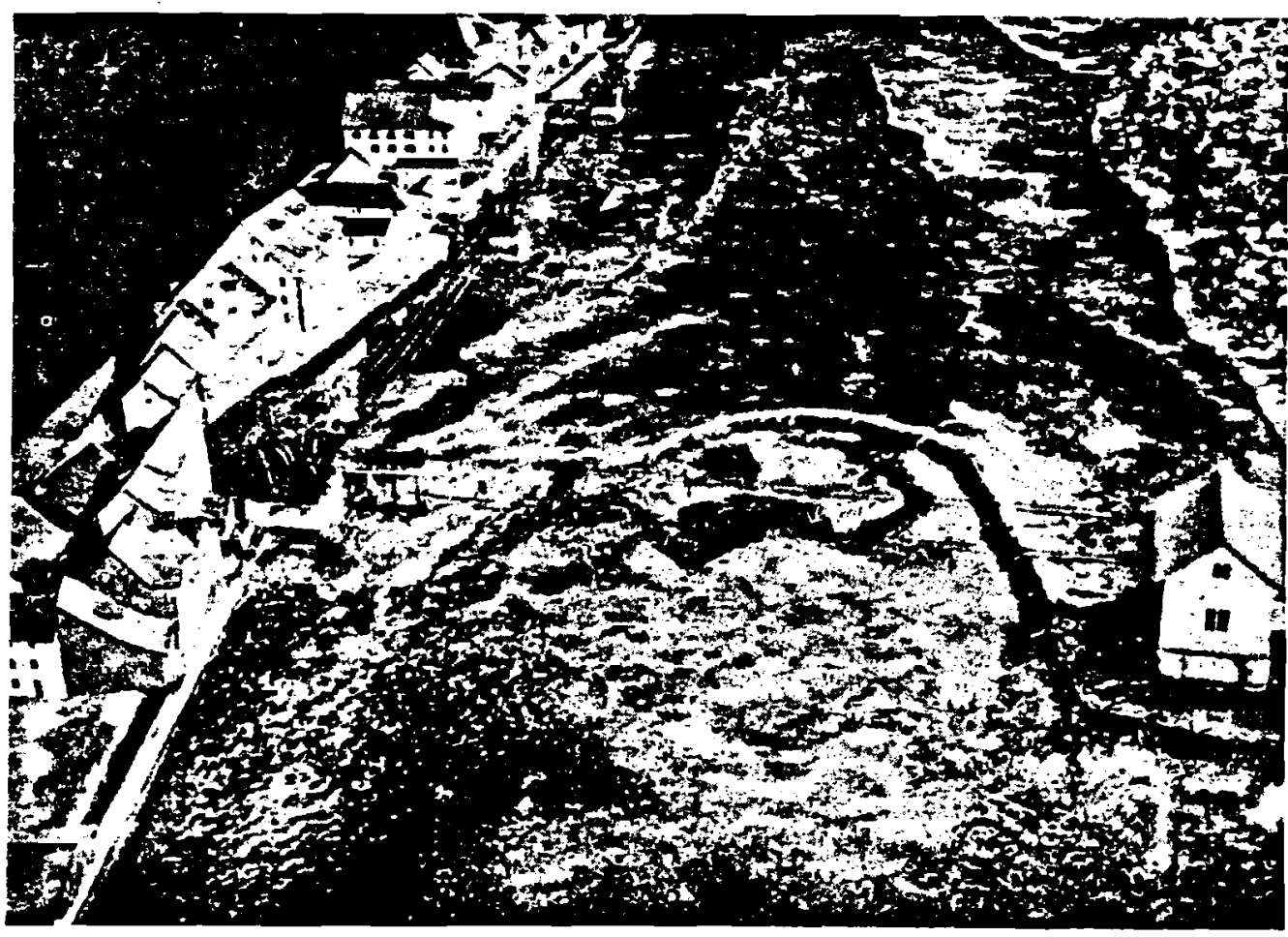

FIc. 4. Cable transportation of mechanical energy-Schafthausen 1866.

hydrogen. carbon monoxide and inerts. Methane was first used to implement the production of this gas, and finally substituted it. As we are travelling back from the consumer to the intermediate system serving him, we should analyse now the problem of the primary source. Our substititution model shows that nuclear energy is the best bet for the next round. The model itself requires perhaps another ten years of data for a precise determination of the slope of the penetration curve. Excluding as very improbable the case that all nuclear programs will be stopped two things can be predicted with a small degree of fuzziness:

- nuclear energy will saturate the electric base load and some of the intermediate load in the late nineties

- natural gas market penetration at the world level will start saturating at the turn of the century.

Both things point to a "call from the market" for a technology capable of interfacing nuclear reactors with a hydrogen system, i.e. to produce hydrogen, presumably by thermal watersplitting. using nuclear heat. as it will be explained in a moment.

For what cuncerns the solution of the technical problem. teeling the call. many bright minds bent over it and one can safely assume by analogy with many similar cases, that if the market will keep calling and support the call with a substantial support, the technologist will find the proper solution. With some pre-knowledge of the papers that will be presented at the First Worlu Hydrogen Energy conference next March, we think the clear signs of convergence toward the objective are already there.

Assuming the problem satisfactorily solved, this "nuclear hydrogen" will naturally seek the most profitable market. This means it will not be piped into the natural gas net to substitute it as 
a fuel but presumably it will displace natural gas trom the plants making hydrogen from it. e.g. for the production of methanol and ammonia. This hydrogen is in fact worth on the market perhaps three times more than natural gas on a caloric basis.

Another high value utilization will probably be in the steel industry. There it may displace coke from the reduction of iron ore as hydrogen can be quite efficiently "meshed" into the blast furnace cycle to reduce coke consumption [6].

When studying the dynamics of systems one has to look for the driving forces. We think in this case these forces are located inside the nuclear industry, and will try to expose our argument.

Assuming that nuclear technology will penetrate the electric markel following a logistic curve as all technologies seem to do [1,2], Fig. 3, and assuming the late nineties witnessing the saturation of that market as our initial data for the nuclear penetration indicate, then the middle of the eighties will be the point where the second derivative of the curve changes sign and the nuclear industry will start feeling a change in rate. probably interpreted as a "slack". The normal reaction in such cases is to try to extend the market by differentiating it. and making hydrogen for the already existing industrial hydrogen market will appear as the obvious solution, and the prize will be huge for the ones that will be ready.

The long range consequences of this sideline operation can be momentous.

First the disengagement from the old fuels can proceed to the full substitution. This does not mean a direct competition in the medium term. As our model shows phasing our primary energies are not influenced by newcomers. Contrary to current literature and intuition nuclear energy is not substituting coal in power stations. Coal would have been substituted by oil and gas anyway, a trend that started in US long before the appearance of nuclear energy. Nuclear energy in a sense substitutes oil but especially gas that would have substituted coal in its absence.

The current intuition that gives so inuch weight to availability of resource and so little to market trends. may lead to gross errors of evaluation. materializing in growing heaps of unsold coal. e.g. in Germany, in spite of the numerous efforts to bribe the consumers to buy it.

Second the scale of the new system, fostered by the transportability of hydrogen, will call for much larger nuclear reactors and fewer sites. This may lead to the much hoped for decoupling between the energy centers and the sociosphere. today made practically impossible hy the peu: transportability of eiectricity which call tor relative small. and numerous reactors not too far from the consumption centers. Unhappily. the new trend will come too late as the nuclear electric power stations will already be built. It could be a sign of foresight to build them as tar as possible on barges so that redeployment will be possible following a rethinking of the system. Also the final disposal of the reactors, a problem lightly skipped at present. may profit from a larger set of solutions if reuctors were transportable over the ocean.

Third. the very large scale of the nuclear reactors that uptimize systems based on a highly transportable intermediate like hydrogen. will lead to great economies of scale, as the nuclear island. i.e. the reactor up to the heat exchangers with related machinery and buildings. appears to have a sciale factor of 0.4 [7]. As we lave said betore. discussing energy systens. the uptimization of a hydrogen net may call for generating stations one or two orders of magnitute larger than an electric unit.

The visualization of such gigantism produces startled reactions from the experts. but past experience shows that technology is apt to produce almost anything when there is a strong and persistent demand. After all electric generators have increased in capacity by five orders of magnitute in only 80 years.

Figure 5 shows the analogous case of supertankers whose rapid increase in tonnage (doubling every 6 vears) was evidently called in by the development of oil trade and not. as the current intuition suggests. by the intrinsic development of tanker technology.

Fourth. as hydrogen is easily storable [10] in various ground structures, exhausted gas fields and aquifers in particular, the plants and most of the trunklines can work around the clock making utilization coincide with availability

Putting these things together, the investment per unit of energy delivered to the consumer can be an order of magnitute lower than in the case of an electric system [8]. With the energy systems becoming more and more capital intensive the attractiveness of hydrogen may be hardly resisted. 


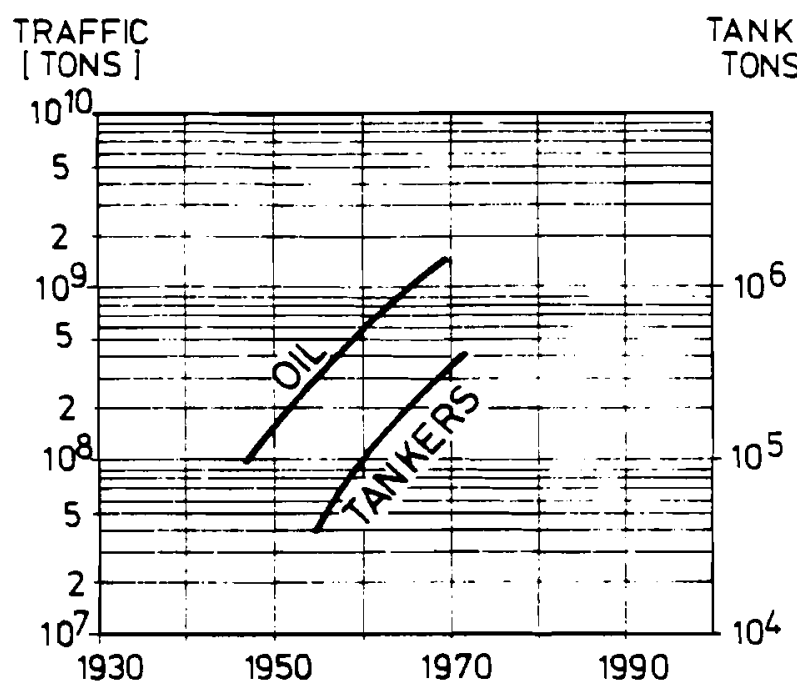

FiG. 5. Correlation herween size of tankers and Oil Tratic.

\section{ON THE PRIMARY ENERGY SOURCES}

As we have seen. hydrogen "downstream" mav first develop a transportation system of its own to penetrate the chemical and metallurgical market and tinally slip into that already developed for natural gas. It will be interesting to see how flexible can be "upstreain" in tapping the new energy sources that may eventually rise over the horizon. Electrical systems did already show to te healthily omnivorous.

Fusion. At first sight tusion reactors appear suitable to produce the $800-100()^{\circ} \mathrm{C}$ which seem necessary for efficient thermochemical watersplitting. More ad hoc processes may he envisaged [9]. The economies of scale of these reactors are not known but present day conceptual designs show large scale being somehow necessary. As a hydrogen system in the first decades of the next century might be able to accept unit powers of the urder of $100 \mathrm{GWth}$. the next technology will be able to choose any size intrinsically convenient.

Solar. Miniequipment to heat the house or similar will presumably not protit from a hydrogen technology. Some ways of storing heat transporting hydrogen in and out metallic hydrides have been proposed. however.

Medium plants. at the beginning, can probably produce electricity and make use of some of the elasticity inherent to the existing electrical nets to take care of the periodicity and vagaries of their output.

If the technique spreads, the numerous medium plants and the few large plants located in remote regions will almost certainly need hydrogen to take the tunction of storage and provide transportability. The processes to split water can be the same as before in case concentrators are used. But again at hoc processes can be developed taking advantage of the physical characteristics of this energy [11].

Similar considerations are valid for wind energy and for ocean thermal energy plants. In the last case hydrogen will provide transportability. perhaps in form of ammonia.

Seen from a distance the technical picture appears all bright, hydrogen having the potential to repeat on a broader scale the feats of electricity during this century. But what about the social aspect of the operation?

The most irritating aspect of the large scale use of fossil fuel has always been pollution. People periodically react to this and the last upheaval has induced the introduction of constraints in their use that will finally lead to ridiculous situations if the pollution has to be reduced in spite of increasing energy consumption. 
Hydrogen. if properly used, will completely solve the problem of chemical pollution at the root. intrinsically, i.e. without technological appendages if a non-fossil primary energy is used for its production.

In the case that for some reason humanity will stick to the old fossil fuels till exhaustion. or new ones, e.g. tar sands, are going to be developed, a fuel cycle can be conceived, similar in principle to that of nuclear reactors, where the primary fuel is used to produce hydrogen, and all "waste" products including $\mathrm{CO}_{2}$ is properly disposed of. The scheme appears feasible engineering and economic wise, and is under consideration at IIASA. In this way the long term climatic effects of $\mathrm{CO}_{2}$, if real, could be avoided by a proper technological fix leading to hydrogen again as the main energy vector to be distributed to the final consumer.

Apart from the chemical, visual pollution will be eliminated as the generating plants would be very remote. transportation and distribution made with buried pipelines. The nasty traffic of trailers and trucks carrying oil products (again the consequence of their poor transportability at the level of the final distribution) can be eliminated. Car and planes can be adapted to use hydrogen and current developments are very promising [5]. The air transport system may even adopt hydrogen internally, i.e. independently from the development of a hydrogen system, just because of the intrinsic advantage of this fuel [5].

\section{CONCLUSION}

An energy system based on hydrogen as energy vector appears the best successor of clectric systems perfectly matching the characteristics and constraints of the energy market including the social constraints and the characteristic of the emerging energy sources.

A long term solution is then available to greatly extend energy use. for which poor countries wiil tinally benefit most, without wretching our standard of living through the backdoor of secondary effects.

\section{REFERENCES}

1. J. C. Fishter \& R. H. PRY. A simpie substitution model of technological change, G. E. Report $7(1-C \cdot 21.5$ (June 1970$)$.

2. R. H. Pry. Forecasting the diffusion of technology, G. E. Report 73-CRD-220 (July 1973).

3. C. MARChETt. Primary energy substitution model. Chem Econ. Engin. Rev. 7, 9(1975).

+ C. MaRchETTI. Transport and storage of energy. IIASA Research Report RR-75-38 (November (475). Also in the proceedings of the Third General Conference of the European Physical Societv. Bucharest. September 9-12 (1975).

$\therefore$ T. V. V'rikocilul (Ld.), Hydrogen Encrg!: Plenum Press, New York (1975).

h. J. Astil: H. Della CaSA \& R. Jon, Conception d"une usine siderurgique, Conference on Hydrugen. Energy. Pollution. Suc. Ing. Civil de France. Paris. May 9 (1974).

7. Private inforniation from General Electric and Westinghouse. See e.g. WANL-2445-1 for sensitivity studies on Very High Temperature Reactors.

ฬ. Private communication from a study by Electricité de France.

9. B. E.Astllinis \& W. C. Gough. Generation of Hydrogen by U. V. Light Produced by the Fusion Torch. 163 Nat. Meeting Am. Chem. Soc., Boston (1972).

11. (. Marcherli. Hydrogen and Energy, Chenl. Econ. Engin. Rev. 5, 7 (1973).

11. A. Fluhina \& K. Hondoa. Valure. 238, 37 (1972). 